\title{
Repercusión de los rankings universitarios en la prensa española
}

\author{
Ma Guadalupe González-Riaño*, Rafael Repiso**, Emilio Delgado López-Cózar*** \\ * Grupo de Investigación EC3. Correo-e: lupegg@movistar.es \\ ** Universidad Internacional de la Rioja. Grupo de investigación EC3 \& EC3metrics. Correo-e: rafael.repiso@gmail.com \\ *** Universidad de Granada. Grupo de Investigación EC3 \& EC3metrics. Correo-e: edelgado@ugr.es
}

Recibido: 14-10-2013; 2a versión: 08-01-2014; Aceptado: 23-01-2014.

Cómo citar este artículo/Citation: González-Riaño, M. G.; Repiso, R.; Delgado López-Cózar, E. (2014). Repercusión de los rankings universitarios en la prensa española. Revista Española de Documentación Científica, 37(3):e055. doi: http://dx.doi. org/10.3989/redc.2014.3.1128

Resumen: La proliferación de rankings de universidades en las últimas décadas y su papel emergente en la evaluación de los sistemas universitarios justifican el estudio de estos productos en los medios de masas. El presente trabajo pretende estudiar la repercusión que han tenido los ranking de universidades en la prensa española durante 160 meses, periodo comprendido entre el 1 de enero de 1998 hasta el 30 de abril de 2012, para ello se ha utilizado MyNewsOnline $®$. Se analiza la producción temporal, territorial y por cabeceras. Igualmente se estudian las noticias que han generado los principales rankings de universidades. Los resultados demuestran el creciente interés de los medios por estas herramientas científicas, especialmente por parte de la prensa de ámbito nacional. En las cabeceras de cobertura nacional destacan los rankings ARWU y Times, mientras que en la prensa local lo hace el Scimago. Las cabeceras más productivas son El País, La Vanguardia y $A B C$.

Palabras clave: Ranking de universidades; educación superior; medios de comunicación; periódicos; noticias.

\section{The impact of university rankings in the Spanish press}

Abstract: The proliferation of university rankings in the last few decades and their increasing role in the evaluation of university systems justify the study of their impact on the media. The present work aims to measure the impact of university rankings in the Spanish press over 160 months, between January 1, 1998 and April 30, 2012. Using the news database MyNewsOnline as the main source of information, we analysed the production of news according to the following variables: timing, geographical distribution, newspaper and rankings. Results show an increasing interest in these tools. The Shanghai Ranking and Times Higher Education University Ranking stand out in the national press, while in the local press the Scimago Institutions Ranking draws the most attention from media. The newspapers with the most news about rankings are "El País", "La Vanguardia" and "ABC".

Keywords: University Rankings; Higher Education; Mass Media; Newspapers; News.

Copyright: () 2014 CSIC. Este es un artículo de acceso abierto distribuido bajo los términos de la licencia Creative Commons Attribution-Non Commercial (by-nc) Spain 3.0. 


\section{INTRODUCCIÓN}

Las universidades, las instituciones que desde su nacimiento en el siglo XI en el occidente europeo asumieron la misión de transmitir la alta cultura y la formación para el ejercicio de las profesiones intelectuales (Ortega, 2007), que más adelante asumieron la función de generar la investigación (siglos XIX y XX), y de transmitir el conocimiento a la sociedad siendo fuentes de innovación y desarrollo (siglo XXI), se han convertido en un recurso estratégico para los países. Son un factor clave para el desarrollo socioeconómico de una nación; su auténtico motor y la clave para su desarrollo futuro, por lo que actualmente se utiliza como un indicador añadido para medir la competitividad global de las naciones (Hazelkorn, 2012a).

Ante esta constatación no es de extrañar la aparición y multiplicación de universidades en todos los países del mundo (se calculan unos 15.000 centros según indica la International Association of Universities, 2012). Este fenómeno, signo de la globalización de la enseñanza superior, ha desatado una competencia feroz entre universidades, no sólo por conseguir recursos económicos (financiación pública y privada) (Ortega, 2009) sino por atraer a los mejores profesores y estudiantes.

De ahí la necesidad que sienten las universidades de demostrar ante la sociedad en su conjunto, y muy especialmente a estudiantes, profesores, gestores y responsables de políticas públicas y empresas, la excelencia en la prestación de sus servicios. Es, justamente, respondiendo a esta necesidad el por qué surgen los rankings de universidades (Aguillo y otros, 2010; Delgado López-Cózar 2012, Orduña 2012), cuyo desarrollo y expansión han sido prolijamente descritas por Orduña (2012). La fatal atracción hacia los rankings universitarios, como era calificada por Van Raan (2005), a la que estamos especialmente predispuestos en las sociedades modernas, se fundamenta en que dichos rankings al ofrecer datos sobre las distintas dimensiones de una universidad (docencia, investigación, transferencia, gestión e infraestructura), al visualizarse en forma de listado nos genera un compendio de la realidad conocida (Eco, 2009) y al, estar sometida a un orden, nos ofrece una visión comparativa entre los elementos descritos en la lista (Repiso y otros, 2013).

Los ranking universitarios contribuyen a construir una imagen de las universidades de extraordinario impacto en la sociedad que van más allá de los simples resultados que proporcionan (Marginson y Wende, 2007; Rauhvargers 2011). Los rankings son herramientas, que por su simplicidad, llegan fácilmente al público general y comienzan a ser claves para cuestiones tan concretas como la decisión de dónde estudiar y, a partir de ahí, influir en la representación que el ciudadano se hace del sistema universitario. Así no es de extrañar que las universidades empiecen a ser juzgadas y valoradas por su posición en estos rankings
(Hazelkorn, 2007), e incluso que dichos rankings estén construyendo la realidad y que se estén empleando de hecho para comparar el prestigio de los países y su importancia en el orden mundial (Hazelkorn, 2012b).

Por este cúmulo de razones, los rankings de universidades han proliferado en todos los países del mundo (no menos de 40 países publican regularmente uno o más rankings http://www.ihep.org/ Research/Rankingsystemsclearinghouse.cfm). Aunque es justo reconocer que la relevancia de los rankings universitarios se hizo especialmente patente desde junio de 2003, cuando profesores de la Shanghái Jiao Tong University (SJTU) elaboraran el "Academia Ranking of Word Universities" (ARWU), que ha provocado que la inclusión o exclusión en el también conocido como "Ranking de Shanghái" cree una jerarquía de facto en el ámbito universitario sin precedentes (Marginson y Wende, 2007). A partir de ese momento el número de rankings se incrementa exponencialmente, naciendo algunos de los rankings globales más conocidos (THE World Universities Rankings, QS University Rankings, Leiden Ranking, NTU Ranking más conocido como "Ranking de Taiwán"). En esta eclosión de rankings España ha jugado un papel protagonista, ya que varios de los rankings más prominentes a nivel mundial se han confeccionado en nuestro país. Es el caso del "Webometrics Ranking of World Universities" (Aguillo, 2012) publicado por el Cybermetrics Lab del CSIC, o el Scimago Institutions Ranking elaborado por el grupo Scimago; a los que hay que añadir el Ranking IUGR de Universidades Españolas por Campos y Disciplinas Científicas elaborado por los grupos EC3 y SCI2S de la Universidad de Granada, ya que como indica Elías Sanz y otros (2013) la existencia de rankings nacionales "resulta esencial para las regiones y países no centrales".

Toda esta proliferación de rankings provoca una avalancha de comunicados y noticias sobre rankings que pasan de "producto de consumo" a "instrumento estratégico global" (Hazelkorn, 2012a). No es de extrañar, por consiguiente, que los medios de comunicación, los transmisores y cristalizadores de la información circulante en la sociedad, también se hayan sentido atraídos por los rankings. La sociedad demanda información y los medios de comunicación hacen visibles los rankings al acercarlos a la ciudadanía y a las universidades, lo que acrecienta un impacto. Se trata además de una información muy mediática (Delgado López-Cózar 2012). Ya en 1910 el listado de universidades elaborado por la American Association of Universities (AAU) fue filtrado y publicado en la prensa norteamericana (Myers y Robe, 2009; Stuart, 1995) citado en (Orduña 2012).

A la vista del interés despertado entre los lectores por este tipo de información, los propios medios se han convertido en productores de rankings. Posteriormente, en 1957, el periodista Chesly 
Manson publica en el Chicago Sunday Tribune una clasificación de universidades, primera lista de universidades creada por un medio (Orduña, 2012); pero el espaldarazo definitivo vendrá en 1983 con la aparición del US News and World Report, que ha convertido su ranking en un negocio sumamente rentable (Van Dyke, 2005). No sorprende que otros periódicos se hayan embarcado en iniciativas parecidas (Financial Times, The Economist, The Wall Street Journal, El Mundo...) que en muchos casos, como el admitido por U.S. News Magazine, les reporta millones de visitas.

Como derivado de esta situación, entendemos que podría ser de interés determinar la repercusión mediática de los rankings de universidades, aspecto que no ha sido indagado hasta el presente con estudios empíricos. Dado el protagonismo español en la generación de rankings, hemos limitado el ámbito de seguimiento a la prensa española. El objetivo de este trabajo es describir las noticias que se han publicado en la prensa española sobre rankings de universidades. Esto nos permitirá obtener una visión general del impacto que los rankings han tenido y tienen en la prensa española, así como ver cuál es la distribución de noticias por cabeceras, temporalización (años, meses y días) y la presencia de los diferentes rankings en estas noticias.

\section{MATERIAL Y MÉTODOS}

Estudio descriptivo longitudinal retrospectivo de las noticias producidas sobre rankings universitarios en los principales periódicos españoles desde el 1 de enero de 1998 hasta el 30 de abril de 2012 .

Para conformar el corpus del estudio se ha tomado como fuente de análisis las noticias producidas en la prensa española y registradas en la base de datos MyNewsOnline $\AA$. MyNewsOnline ${ }^{\circledR}$ es una base de datos que indiza más de 200 periódicos y que contiene más de 120 millones de artículos en castellano, catalán, euskera y gallego. De origen comercial y no académico, es el sistema de información de más amplia y exhaustiva cobertura de noticias de prensa españolas (Grau y Guallar, 2004) que ya ha sido utilizado en investigaciones académicas previas (Repiso y otros, 2013). No obstante, conviene advertir que no todos los periódicos están completamente indizados durante todo el periodo analizado por razones contractuales. MyNewsOnline, base de datos de actualización diaria, comienza a indexar periódicos en el año 1995. El estudio comienza en 1998, el año en que aparecen las primeras noticias sobre rankings universitarios en la prensa española indizada en esta base de datos.

A fin de asegurar la máxima exhaustividad, se aplicó una estrategia de búsqueda que contemplara una consulta general sobre los rankings de universidades y las propias para cada ranking para recuperar todas las noticias publicadas sobre ellos (Tabla I). La búsqueda y recopilación de las noticias se realizó el 30/04/2012, y la ventana temporal de la búsqueda comprendió desde el 01/01/1998 hasta el mismo 30/04/2012. Dado que la mayoría de los rankings estudiados surgen a partir de 2004, convenía tomar un período de tiempo amplio (anterior y posterior a dicha fecha) que permitiera calibrar adecuadamente la repercusión de los rankings.

Se recuperaron 1123 noticias de 82 periódicos, que, tras la eliminación de duplicados y la normalización de los registros, quedaron reducidas a 810 noticias. Los resultados se depuraron para sólo dejar medios españoles con edición nacional (16 periódicos), regional (43 periódicos) y local (40 pe-

Tabla I. Ecuación de búsqueda para la recuperación de noticias sobre ranking universitarios

\begin{tabular}{|c|c|}
\hline Temática & Términos de búsqueda \\
\hline Ranking de universidades & (Ranking and Universidades) \\
\hline Ranking de shangai/shanghai/ARWU & Ranking AND (Shangai OR Shanghai OR ARWU) and Universidades \\
\hline Ranking ISI & "Ranking ISI" \\
\hline Ranking Web de Universidades & Webometrics OR "Ranking web de universidades" OR (Ranking and CSIC) \\
\hline THE World Universities Rankings & "THE World Universities Rankings" OR "Ranking and Times and Universidades" \\
\hline Ranking de Taiwán & Ranking AND Taiwán AND Universidades \\
\hline Ranking Scimago & Ranking AND Scimago \\
\hline Ranking de Leiden & Ranking AND Leiden \\
\hline
\end{tabular}


riódicos). Del total de las 810 noticias obtenidas de MyNewsOnline ${ }^{\circledR}$ hay que tener en cuenta que proceden de medios en los que en la mayoría sigue vigente su cobertura (a excepción de Deia que no está desde enero del 2012 y El País Internacional S.A., que se dio de baja en enero del 2005, pero El País en su edición nacional sigue vigente).

\section{RESULTADOS}

Durante los 160 meses que abarca el periodo analizado (1/1/1998-30/40/2012) se han publicado un total de 810 noticias sobre rankings de universidades en la prensa española, identificadas en la base de datos MyNewsOnline $\AA$. Analizando la evolución de las noticias publicadas puede apreciarse un incremento significativo a partir de 2007. Si en el septenio 1998 y 2004 la edición de noticias sobre ranking de universidades está por debajo de las 20 por año, y en el bienio 2005-2006 pasa a las 30 , en 2007 se incrementa a las 65, superando las cien en 2008 y 2009. En el año 2010 se registra el punto más elevado con 191 noticias y en 2011 baja hasta las 131, situándose no obstante en el segundo año por producción (Figura 1).
Hasta 2006 el número de noticias aparecidas en la prensa local, regional o nacional sigue una evolución muy similar. En cambio, entre 2007 y 2009 se dispara el número de noticias publicadas en prensa nacional, fenómeno que se invierte entre 2010 y 2011 cuando son las noticias aparecidas en la prensa local y regional las que más abundan. Los medios nacionales muestran altibajos aunque concentran el mayor volumen entre 2008-2011 y los regionales son más arrítmicos, no superan las 30 noticias en el trienio 2007/08/09, pero repuntan hasta el pico más alto en 2010 con 73 noticias. Así, la distribución por ámbito en 2010 estuvo igualada entre los medios regionales (con 73 noticias) y locales (con 72), mientras que los nacionales registraron sólo 46 noticias.

La cobertura de las noticias sobre rankings de universidades durante los meses del año es variada (Figura 2). Es en octubre cuando se registra el mayor número de noticias, seguido de septiembre y noviembre. Los meses que registran menor número de noticias son julio y agosto, el periodo universitario vacacional. En relación con la distribución de noticias por días parece claro que es el inicio de la semana el periodo más usado por los medios para difundir las noticias sobre rankings.

Figura 1. Distribución de noticias publicadas sobre rankings de universidades en prensa española por años y audiencia geográfica

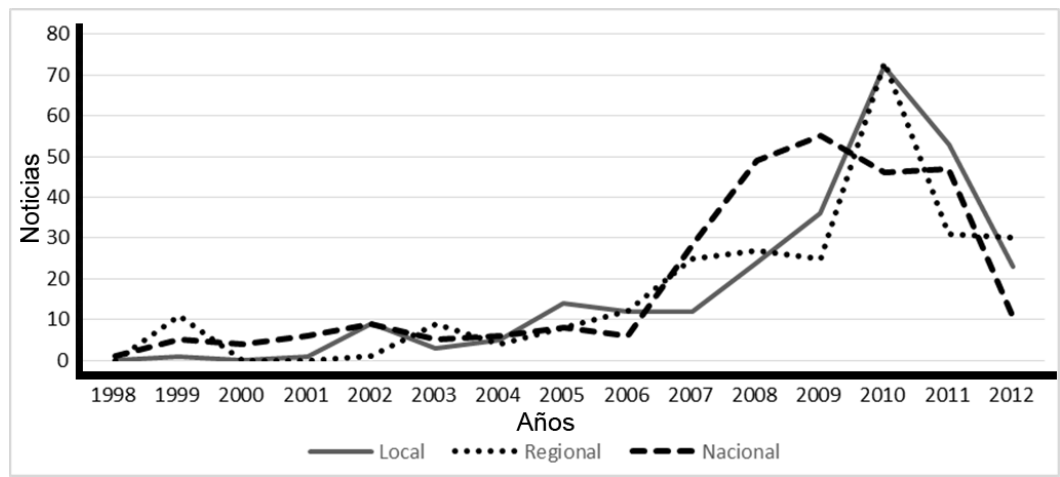

Figura 2. Distribución de noticias publicadas en la prensa española sobre rankings universitarios por meses y días
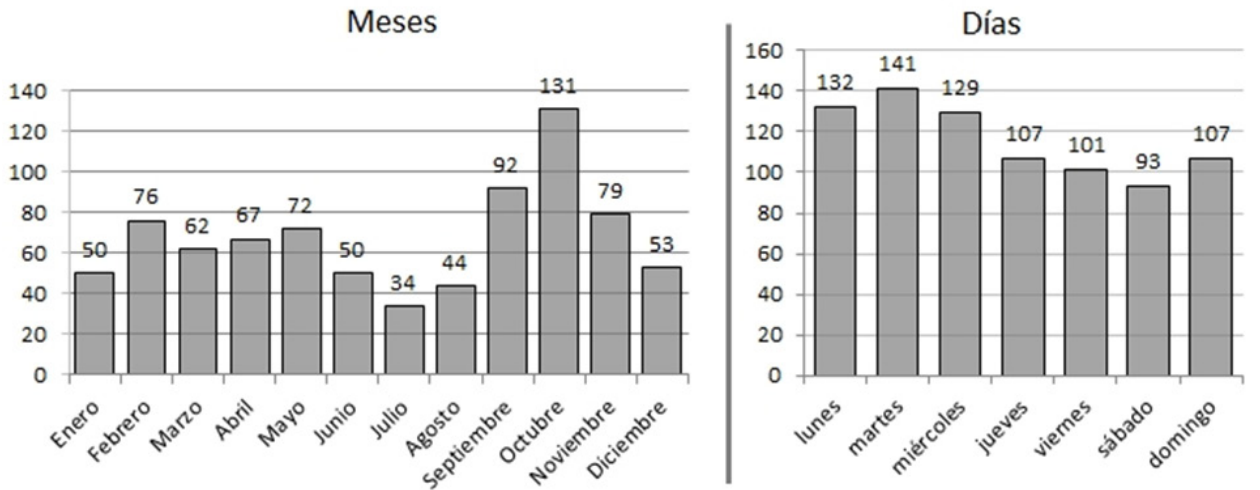
Respecto a cuales son los rankings que reciben una mayor atención de la prensa española, cabe destacar al Ranking Shanghái pues concentra más de un tercio del total de las noticias (Tabla II). El Ranking Scimago, de ámbito internacional elaborado en España, ocupa el segundo lugar con 240 registros y supera al Ranking Times (THE) (considerado el segundo de ámbito mundial/global que comienza en 2004) y que genera 182 . Otro ranking internacional y también de elaboración nacional, el denominado Webometrics que aparece en 2004, ocupa el cuarto lugar con 98 noticias seguido del nacional Ranking I-UGR con 31 registros. Los dos que menos noticias acaparan son mundiales; el Ranking de Taiwán y el Leiden Ranking que tienen 17 y 7 noticias recuperadas, respectivamente.

Si analizamos geográficamente en qué tipo de prensa aparecen publicadas más veces noticias sobre los rankings, observamos (Tabla III) que el denominado ranking de Shanghái lidera el mayor número de noticias publicadas en prensa nacional (134 noticias) y regional (92), aunque es desbancado por el Ranking Scimago en el ámbito local (150 noticias). El Ranking Times se sitúa en cuarto lugar con 103 noticias en la prensa nacional.

Ahora bien, la disparidad informativa entre lo más cercano y lo general se observa al sumar los registros totales de local y regional. En el caso del Ranking de Shanghái las 202 noticias (local más regional) superan a las nacionales (134) y lo mismo ocurre con las noticias del Ranking Scimago (203) respecto a las 37 nacionales y en el Ranking I-UGR (27) respecto a 4 nacionales. Por el contrario el Ranking Times tiene más noticias nacionales (103) aún uniendo las regionales y locales (79) al igual que el Ranking Webometrics cuyo número está más igualado (50 nacionales y 47 regionales y locales).

Tabla II. Evolución del número de noticias de rankings universitarios en la prensa española (2000-2012)

\begin{tabular}{|c|c|c|c|c|c|c|c|c|}
\hline Años & Shanghai & Scimago & Times & Webometrics & I-UGR & Taiwán & Leiden & Total \\
\hline 2000 & & & $100,00 \%$ & & & & & 1 \\
\hline 2001 & & & $100,00 \%$ & & & & & 2 \\
\hline 2002 & & & $100,00 \%$ & & & & & 1 \\
\hline 2003 & & & $100,00 \%$ & & & & & 5 \\
\hline 2004 & $66,67 \%$ & & $33,33 \%$ & & & & & 6 \\
\hline 2005 & $45,45 \%$ & $13,64 \%$ & $18,18 \%$ & $22,73 \%$ & & & & 22 \\
\hline 2006 & $53,33 \%$ & $23,33 \%$ & $20,00 \%$ & & & $3,33 \%$ & & 30 \\
\hline 2007 & $44,62 \%$ & $13,85 \%$ & $26,15 \%$ & $13,85 \%$ & & $1,54 \%$ & & 65 \\
\hline 2008 & $51,72 \%$ & $1,15 \%$ & $24,14 \%$ & $19,54 \%$ & $1,15 \%$ & $1,15 \%$ & $1,15 \%$ & 87 \\
\hline 2009 & $27,47 \%$ & $20,88 \%$ & $27,47 \%$ & $21,98 \%$ & & $1,10 \%$ & $1,10 \%$ & 91 \\
\hline 2010 & $43,84 \%$ & $24,92 \%$ & $13,81 \%$ & $12,61 \%$ & $2,10 \%$ & $2,70 \%$ & & 333 \\
\hline 2011 & $36,17 \%$ & $15,60 \%$ & $33,33 \%$ & $1,42 \%$ & $10,64 \%$ & $2,13 \%$ & $0,71 \%$ & 141 \\
\hline 2012 & $10,00 \%$ & $73,85 \%$ & $3,85 \%$ & $2,31 \%$ & $6,15 \%$ & $0,77 \%$ & $3,08 \%$ & 130 \\
\hline Total & 339 & 240 & 182 & 98 & 31 & 17 & 7 & 914 \\
\hline
\end{tabular}

Tabla III. Presencia de noticias en prensa sobre rankings de universidades por ámbito territorial

\begin{tabular}{lccc}
\hline & Local & Regional & Nacional \\
\hline Shanghai & $34,48 \%$ & $37,40 \%$ & $38,84 \%$ \\
\hline Scimago & $47,02 \%$ & $21,54 \%$ & $10,72 \%$ \\
\hline Times & $5,33 \%$ & $25,20 \%$ & $29,86 \%$ \\
\hline Webometrics & $8,46 \%$ & $8,13 \%$ & $14,49 \%$ \\
\hline I-UGR & $3,45 \%$ & $6,50 \%$ & $1,16 \%$ \\
\hline Taiwán & $0,31 \%$ & $0,41 \%$ & $4,35 \%$ \\
\hline Leiden & $0,94 \%$ & $0,81 \%$ & $0,58 \%$ \\
\hline
\end{tabular}


Los resultados sobre el número de noticias registradas por periódicos, indican que El País, La Vanguardia y $A B C$ son los medios que ocupan las tres primeras posiciones. El periódico que lidera el mayor número de noticias publicadas (Tabla IV) es el medio de edición nacional El País (72) seguido por un medio regional La Vanguardia (63) y el tercer y cuarto puesto lo ocupan medios nacionales, el $A B C$ (50) y El Mundo (46). El quinto lugar lo ocupa el regional La voz de Galicia (33) al que siguen en los puestos sexto y séptimo dos medios especializados en economía: La Gaceta de los Negocios (31) y Expansión (28). Entre los diez primeros hay cuatro diarios locales, tres de ellos andaluces, que son: Ideal (28), Granada Hoy (22) y El Diario de Sevilla (19) y el diario Las Provincias de Las Palmas (19).

En el análisis por grupo editorial, el Grupo Prisa continuaría en el primer puesto (con 89 noticias al sumar las de El País y las del Cinco Días, que alcanza las 17) seguida del Grupo Vocento (con 78 al sumar las del $A B C$ e Ideal).

Tabla IV. Distribución de noticias sobre ranking universitarios por cabecera de periódicos

\begin{tabular}{|c|c|c|c|c|c|c|c|c|c|}
\hline POS. & DIARIO & NOTS. & COBERTU & RA & POS. & DIARIO & NOTS. & \multicolumn{2}{|c|}{ COBERTURA } \\
\hline 1 & El País & 72 & ene-98 & - & 41 & Ultima Hora & 6 & may-03 & - \\
\hline 2 & La Vanguardia & 63 & ene-98 & - & 42 & 20 Minutos & 5 & may-06 & - \\
\hline 3 & $A B C$ & 50 & ene-98 & - & 43 & Diari de Tarragona & 5 & nov-01 & - \\
\hline 4 & El Mundo & 46 & ene-98 & - & 44 & Diario de León & 5 & may-01 & - \\
\hline 5 & La Voz de Galicia & 33 & abr-01 & - & 45 & El Periódico de Cataluña & 5 & oct-02 & - \\
\hline 6 & La Gaceta de los Negocios & 31 & jul-97 & - & 46 & La Opinión de Tenerife & 5 & - & - \\
\hline 7 & EXPANSION & 28 & feb-96 & - & 47 & El Segre & 5 & ene-98 & - \\
\hline 8 & Ideal & 28 & oct-02 & - & 48 & Diario de Mallorca & 4 & abr-04 & - \\
\hline 9 & Granada Hoy & 22 & oct-05 & - & 49 & Información & 4 & abr-04 & - \\
\hline 10 & El Diario de Sevilla & 19 & oct-01 & - & 50 & Noticias de Gipuzkoa & 4 & - & - \\
\hline 11 & Las Provincias Las Palmas & 19 & abr-04 & - & 51 & ADN & 3 & jul-06 & - \\
\hline 12 & La Verdad de Murcia & 18 & abr-03 & - & 52 & Deia & 3 & feb-12 & ene-12 \\
\hline 13 & Cinco Días & 17 & ene-98 & - & 53 & Diario del Alto Aragón & 3 & oct-03 & - \\
\hline 14 & El Correo & 17 & may-02 & - & 54 & Diario Información & 3 & abr-04 & - \\
\hline 15 & El Diario Vasco & 15 & oct-02 & - & 55 & El Adelanto & 3 & may-01 & - \\
\hline 16 & El Economista & 15 & jun-06 & - & 56 & EL PAIS Internacional, S.A. & 3 & - & sep-05 \\
\hline 17 & El Diario de Córdoba & 13 & oct-01 & - & 57 & La Voz de Cádiz & 3 & nov-05 & - \\
\hline 18 & El Periódico de Aragón & 11 & may-01 & - & 58 & Negocio & 3 & - & - \\
\hline 19 & El Periódico de Extremadura & 11 & may-01 & - & 59 & Público & 3 & - & - \\
\hline 20 & Córdoba & 10 & may-01 & - & 60 & Cataluña Económica & 2 & - & - \\
\hline 21 & Diario Sur & 10 & - & - & 61 & Diari Segre & 2 & ene-98 & - \\
\hline 22 & El Diario de Cádiz & 10 & ene-98 & - & 62 & Diario de Pontevedra & 2 & ene-10 & - \\
\hline 23 & El Norte de Castilla & 10 & abr-03 & - & 63 & El Diario de Baleares & 2 & may-03 & - \\
\hline 24 & El Periódico & 10 & ene-00 & - & 64 & El Faro de Murcia & 2 & oct-09 & - \\
\hline 25 & El Comercio & 9 & may-03 & - & 65 & El Progreso & 2 & ene-10 & - \\
\hline 26 & Huelva Información & 9 & nov-04 & - & 66 & Gara & 2 & oct-02 & - \\
\hline 27 & La Razón & 9 & nov-00 & - & 67 & Heraldo de Aragón & 2 & jul-03 & - \\
\hline 28 & Levante & 9 & abr-03 & - & 68 & La Opinión de Murcia & 2 & abr-04 & - \\
\hline 29 & Diario Noticias Álava & 8 & jun-05 & - & 69 & La Rioja & 2 & may-03 & - \\
\hline 30 & El Correo Gallego & 8 & oct-03 & - & 70 & Presència & 2 & sep-06 & - \\
\hline 31 & El Diario Montañés & 8 & may-03 & - & 71 & Aquí Cantabria & 1 & mar-11 & - \\
\hline 32 & Europa Sur & 8 & oct-01 & - & 72 & Diari Ara & 1 & nov-10 & - \\
\hline 33 & Mediterráneo & 8 & & - & 73 & Diario de Ibiza & 1 & abr-04 & - \\
\hline 34 & Diario de Navarra & 7 & nov-02 & - & 74 & El Global & 1 & may-03 & - \\
\hline 35 & El Diario de Almería & 7 & mar-09 & - & 75 & Estrella Digital & 1 & may-03 & - \\
\hline 36 & Faro de Vigo & 7 & may-03 & - & 76 & La Mañana & 1 & nov-09 & - \\
\hline 37 & Diario de Jerez & 6 & ene-98 & - & 77 & La Opinión de Málaga & 1 & abr-04 & - \\
\hline 38 & Diario Málaga Hoy & 6 & mar-05 & - & 78 & La Voz de Almería & 1 & sep-10 & - \\
\hline 39 & La Nueva España & 6 & abr-04 & - & 79 & Menorca & 1 & mar-09 & - \\
\hline 40 & La Voz de Asturias & 6 & may-01 & - & 80 & QUE & 1 & may-06 & - \\
\hline
\end{tabular}


Un análisis más detallado de los cuatro medios que más publican sobre rankings (concentran 231 noticias de los 810 analizadas) indica que sólo dos de ellos, La Vanguardia y $A B C$, coinciden en registrar mayor volumen de noticias en el año 2010 (Figura 3). En El País se registra el mayor número de noticias en el periodo 2008-2011; La Vanguardia, uno de los primeros en impulsar los suplementos sobre ciencia, manifiesta estabilidad con poca producción y marcados repuntes en 2007 y 2010; $A B C$ oscila con un periodo de incremento entre 2007-2010 y El Mundo (el único que publica la guía de las "50 carreras" desde el curso 2001-2002) comienza con poca producción que aumenta entre el 2008-2011.

\section{DISCUSIÓN Y CONCLUSIONES}

La distribución de noticias por año y ámbitos geográficos de los periódicos que publican noticias sobre rankings universitarios indica la madurez informativa de los rankings que han llegado ya a concitar un interés creciente y sólido en los medios de comunicación. Para los medios de comunicación la publicación de rankings significa un incremento seguro en el número de lectores, como ya atestiguaba el tremendo impacto de la publicación del ranking promovido por el US News and World Report (Delgado López-Cózar, 2012). Indirectamente, refleja el inusitado deseo de los miembros de la comunidad científica y universitaria así como de los gestores y responsables de las políticas universitarias, por este tipo de informaciones que les permiten conocer el impacto y el nivel de sus propios centros de trabajo (Orduña, 2012). Por tanto no es de extrañar que la proliferación de rankings universitarios conlleve a su vez el éxito mediático de los propios rankings.

Los datos analizados referentes a las noticias publicadas en medios de comunicación españoles sobre rankings de universidades entre los años 1998-2012 (hasta abril) indican una progresiva presencia con la cota máxima en el año 2010. Estos resultados son un fiel reflejo de la cronología del nacimiento de los distintos rankings: será en el trienio 2003-2005 cuando aparezcan los principales rankings internacionales (ARWU, THE, SIR, Webometrics), y es justo a partir de 2006 cuando se incremente significativamente el número de noticias publicado en la prensa española.

El incremento de noticias pone de manifiesto la creciente preocupación por la evaluación de las universidades en el entorno político y social español. El debate que se inició previamente a la implantación de los Campus de Excelencia puestos en marcha en el 2008, con sus animadas controversias (Docampo y otros, 2012) contribuyó a que sea el año 2010 el de mayor producción. La posición de las universidades en los rankings mundiales era una información relevante en proceso de internacionalización de las universidades españolas que es el que abría formalmente el programa de Campus de Excelencia. Desde luego los campus internacionales de las universidades españolas fueron noticia. Testimonia este hecho cómo los rankings de universidades se han colado en las agendas de los propios responsables públicos del sistema universitario español. La constatación de que "España no cuenta con ninguna universidad entre las 150 o 200 mejores del mundo en los ranking internacionales" figura en el argumentario de los ministros españoles de educación desde el año 2007.

Las fechas de publicación de noticias sobre rankings se adecúan perfectamente a las fechas de publicación de los distintos rankings y al calendario universitario. En octubre se produce el mayor número de noticias coincidiendo formalmente con el comienzo del curso. No obstante, las noticias se

Figura 3. Evolución de noticias de rankings universitarios por las principales cabeceras

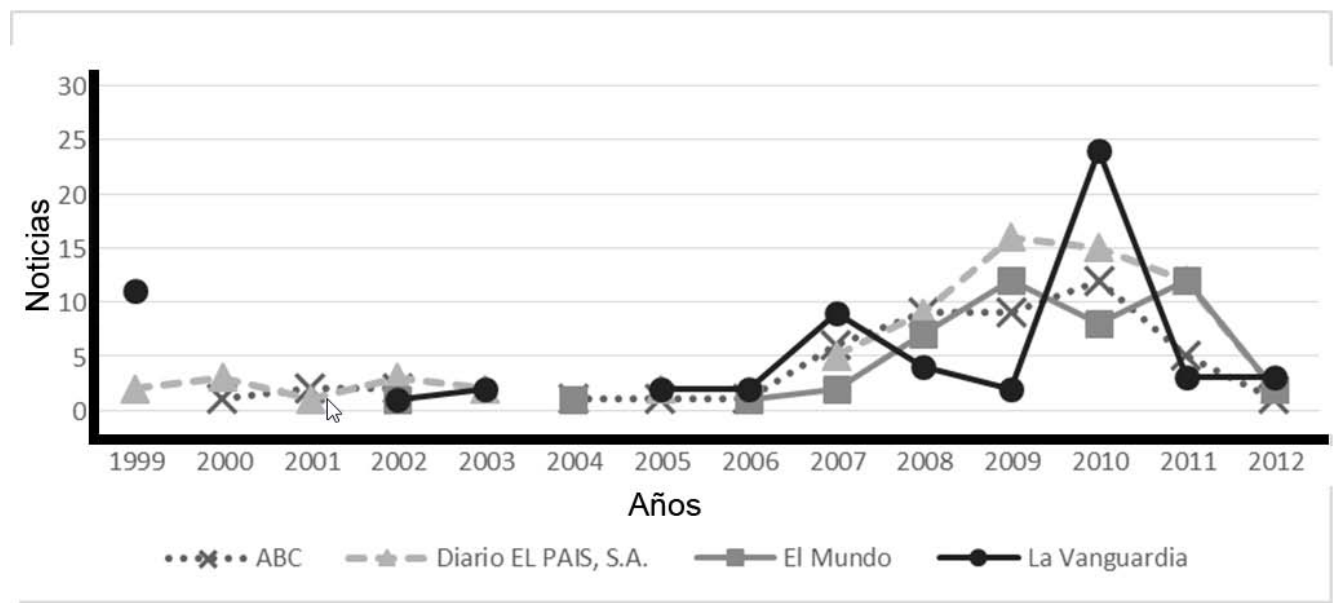


incrementan al mismo ritmo que se van publicando las actualizaciones de los principales rankings internacionales. Por otro lado, los días elegidos para publicar noticias sobre ellos, influenciado por la agenda, se concentra en el inicio de semana, ajustándose perfectamente a la vida académica. De forma general se observa que se producen dos hechos paralelos: los resultados proporcionados en los rankings son esperados y por lo tanto difundidos por los medios en la fecha que se hacen públicos; y a su vez se incrementa el número de noticias al incorporarse nuevos rankings durante los últimos años.

El ranking ARWU y el SIR son los rankings de mayor repercusión mediática, especialmente el elaborado por la Shanghai Jiao Tong University. Es el que más noticias concita globalmente, aunque su presencia se concentra especialmente en los periódicos de tirada nacional y regional, por lo que aglutina una mayor audiencia. En cambio, SIR, es el que recibe más noticias en las cabeceras locales. La razón hay que buscarla en la amplia cobertura de universidades españolas que posee; todas las universidades españolas son cubiertas en el ranking de Scimago, mientras que en el de Shanghái solo se muestran poco más de 10 universidades, que son las capaces de colocarse entre las 500 primeras del mundo. A partir del ranking de Scimago la prensa local siempre puede incluir información sobre la universidad local. En este caso, lo más cercano alcanza más repercusión que lo más lejano. Llama asimismo la atención como dos periódicos granadinos (Ideal y Granada Hoy) se sitúen entre las 10 cabeceras que más noticias publican sobre rankings. No es de extrañar pues en dicha ciudad radican dos de los grupos creadores de estos rankings (SIR de Scimago, y Ranking I-UGR de EC3). Los periódicos locales se convierten en ductil correa de transmisión de la información generada en sus propios entornos universitarios.

Aunque en el análisis no se refleja ni la labor de las agencias de noticias, ni de los gabinetes de comunicación, ni de los suplementos relacionados con la ciencia-educación sí se detecta en los resultados cómo los medios que ocupan los diez primeros lugares como los más leídos son los que más noticias publican sobre ranking universitarios (EI País, El Mundo, La Vanguardia y el $A B C$ ). A ellos se suman los medios locales (tres y andaluces) y los especializados en economía, por lo que se observa un interés desde la prensa, y de todo tipo de prensa, por las noticias sobre rankings. Así cada medio de una determinada comunidad prestará especial atención a los rankings y el contenido vinculado con dicho territorio, quizá en menos noticias con un target mucho más definido. De un ranking "siempre se puede conseguir un buen titular". (Delgado López-Cózar, 2012). Se puede concluir que los rankings poseen una gran atracción mediática, tanto en la prensa nacional pero, sobre todo, en la regional y local. Suelen ocupar un espacio privilegiado entre las noticias de información local.
En definitiva, este estudio demuestra que en los últimos 14 años se han escrito noticias sobre rankings de universidades, que conforma una espiral informativa fomentada por quién los elabora, de cuándo y cómo se hacen públicos, de su difusión en los medios de comunicación y de su interés. Más allá de la credibilidad, de la crítica o del análisis de cómo se elaboran dichos rankings sobre las universidades, queda patente que por su simple publicación impactan tanto en la sociedad, en los gobiernos y en las universidades. Este hecho manifiesta que hoy en día son un elemento a tener en cuenta en función de su capacidad para influir en la determinación la financiación, las políticas educativas, e incluso el futuro que podrían tener las universidades de no lograr los resultados deseados.

\section{BIBLIOGRAFÍA}

Aguillo, I. F.; Bar-Ilan, J.; Levene, M.; Ortega, J. L. (2010). Comparing university rankings. Scientometrics, vol. 85 (1), 243-256. http://dx.doi. org/10.1007/s11192-010-0190-z

Aguillo, I. (2012). Rankings de Universidades: el Ranking Web. Higher Learning Research Communications, vol. 2 (1), 3-12.

CENTRE OF SCIENCE AND TECHNOLOGY STUDIES - UNIVERSITY LEIDEN. Leiden-Ranking. En: http://www.leidenranking.com/ [Accedido el 01/08/2013].

CHE - CENTRUM FÜR HOCHSCHULENTWICKLUNG. CHE Hochschul-Ranking. En: http://www.cheranking.de [Accedido el 01/08/2013].

Delgado López-Cózar, E. (2012). Cómo se cocinan los rankings universitarios. Dendra Médica. Revista de Humanidades, vol. 11 (1), 43-58.

Docampo, D.; Herrera, F.; Luque-Martínez, T.; Torres-Salinas, D. (2012). Efecto de la agregación de universidades españolas en el Ranking de Shanghai (ARWU): caso de las comunidades autónomas y los campus de excelencia. El Profesional de la Información, vol. 21 (4), 428-432. http://dx.doi.org/10.3145/epi.2012.jul.16

Eco, U. (2009). El vértigo de las listas. Barcelona: Lumen.

Gladwell, M. (2011, 14 de Febrero). What College Rankings Really Tell Us: The New Yorker. The New Yorker. http://www.newyorker.com/ reporting/2011/02/14/110214fa_fact_gladwell [Accedido el 10/10/2013]

Grau Moracho, J.; Guallar, J. (2004). My News, la hemeroteca digital de la prensa española. El profesional de la información, vol.13 (6), 466-476. http://dx.doi.org/10.3145/epi.2004.nov.07

Hazelkorn, E. (2007). Are league tables and rankings influencing Higher Education DecisionMaking? IAU Horizons, vol. 13 (2-3), pp. 4.

Hazelkorn, E. (2012a). How Rankings are Reshaping Higher Education. En: Climent, V., Michavila, F. and Ripollés, M. (eds): Los Rankings Univeritarios: Mitos y Realidades. Tecnos. 
Hazelkorn, E. (2012b). Rankings and the Reshaping of Higher Education. The Battle for World-Class Excellence. New York: Palgrave MacMillan 2012.

International Association of Universities (2012). International Handbook of Universities. (250 ed.). London: Palgrave Macmillan.

Marginson, S.; Wende, M. Van der (2007). To rank or to be ranked: the impact of global rankings in higher education. Journal of studies in international education, vol. 11 (3-4), 306-329. http:// dx.doi.org/10.1177/1028315307303544

Myers, L.; Robe, J. (2009). College rankings: history, criticism and reform. Washington: Center of College Affordability and Productivity.

Orduña Malea, E. (2012). Propuesta de un modelo de análisis redinformétrico multinivel para el estudio sistémico de las universidades españolas. Valencia: Universidad Politécnica de Valencia. [Tesis]

Ortega, J. L. (2009). 20. Seminario Internacional sobre Ranking Universitarios. Revista Española de Documentación Científica. vol. 32 (3), 127-129.

Ortega y Gasset, J. (2007). La misión de la Universidad. Madrid: Biblioteca Nueva.

Rauhvargers, A. (2011). Global university rankings and their impact. Leadership for WorldClass UniversitiesChallenges for Developing Countries. Brussels: European University Association.

Repiso, R.; Llorente-Barroso, C.; García-García, F. (2013). Ranking ESCO de agencias de publicidad: Descripción y resultados. El profesional de la información vol. 22 (2), 181-187. http:// dx.doi.org/10.3145/epi.2013.mar.13
Repiso, R.; Rodríguez-Pinto, M. Á.; García-García, F. (2013). Posicionamiento y agrupación de la prensa española: Análisis de contenido de las noticias generadas en la Jornada Mundial de la Juventud 2011. Estudios Sobre el Mensaje Periodístico, vol. 19 (2), 1091-1106.

Sanz-Casado, E.; García-Zorita, J. C.; SerranoLópez, A. E.; Efraín-García, P.; De Filippo, D. (2013). Rankings nacionales elaborados a partir de múltiples indicadores frente a los de índices sintéticos. Revista Española de Documentación Científica, vol. 36 (3). http://dx.doi. org/10.3989/redc.2013.3.1.023

SCIMAGO. SCImago Institutional Ranking (SIR) 2009 World Report. En: http://www.scimagoir. com/ [Accedido el 2/07/2013].

Stuart, D. (1995). Reputational rankings: background and development. New directions for institutional research, (88), 13-20. http:// dx.doi.org/10.1002/ir.37019958803

TIMES HIGHER EDUCATION SUPPLEMENT. World University Ranking Times Higher Education Supplement. En: http://www.timeshighereducation. co.uk/world-university-rankings/ [10/10/2013]

van Dyke, N. (2005). Twenty years of university report cards. Higher Education in Europe, vol. 30 (2), 103-125. http://dx.doi. org/10.1080/03797720500260173

van Raan, A. F. J. (2005). Fatal attraction-conceptual and methodological problems in the ranking of universities by bibliometric methods. Scientometrics, vol. 62 (1), 133-143. http://dx.doi. org/10.1007/s11192-005-0008-6 